\title{
Gasless single-port laparoscopic cholecystectomy
}

\author{
Masashi Ishikawa, Michihito Asanoma, Yoshihiko Tashiro, Hirokazu Takechi, Kazuo Matsuyama, Takayuki Miyauchi \\ Department of Surgery, Shikoku Central Hospital, Shikokuchuo, Japan
}

\begin{abstract}
Purpose: Single-port laparoscopic surgery is anticipated to become the future of minimally invasive surgery. We have devised an alternative approach for laparoscopic cholecystectomy by inserting a single port at the umbilicus and using the abdominal wall-lifting method, without establishing pneumoperitoneum.

Methods: Retrospective analysis of 130 patients undergoing laparoscopic cholecystectomy was done to compare the conventional laparoscopic cholecystectomy $(\mathrm{CLC})(\mathrm{n}=69)$ and the novel single-port laparoscopic cholecystectomy (SLC) using the abdominal wall-lifting method ( $\mathrm{n}=61)$. The surgical procedures were as follows. A 2- to 3-cm transumbilical incision was made, and a wound retractor was inserted into the abdomen without difficulty. Abdominal distension was obtained using a fan-shaped retractor without the use of carbon dioxide insufflations. A 5-mm flexible scope and modified curved graspers and dissectors were used to give the feeling of triangulation during dissection.

Results: The SLC group consisted of 25 males and 36 females with a mean age of $58.1 \pm 7.2$ years and a mean body mass index of $23.1 \pm 3.2 \mathrm{~kg} / \mathrm{m}^{2}$. The two groups were comparable for mean age, sex, disease, American Society of Anesthesiologists physical status classification, and comorbidity. Likewise, the duration of operation, postoperative hospital stays, complications, the number of use of analgesics, and conversion rate to open technique were not significantly different in the two groups.

Conclusion: The impaired view in single-port laparoscopic surgery can be improved by using articulating instruments that can be rotated out of the field of view. This novel gasless method is cost-effective and produces minimal postoperative discomfort with no additional scars.
\end{abstract}

Keywords: Laparoscopic cholecystectomy, Single-incision, Pain

This is an Open Access article distributed under the terms of the Creative Commons Attribution Non-Commercial License (http:// creativecommons.org/licenses/by-nc/4.0/) which permits unrestricted non-commercial use, distribution, and reproduction in any medium, provided the original work is properly cited.

Received May 17, 2021

Revised July 13, 2021

Accepted August 9, 2021

Corresponding author

Masashi Ishikawa

Department of Surgery, Shikoku

Central Hospital, 2233 Kawanoecho,

Shikokuchuo, Ehime 799-0193, Japan

Tel: +81-896-58-3515

Fax: +81-896-58-3464

E-mail: masaishi1122@gmail.com

ORCID:

https://orcid.org/0000-0001-5434-6443
Copyright (C) The Korean Society of Endoscopic and Laparoscopic Surgeons.

\section{INTRODUCTION}

Single-port laparoscopic surgery is a rapidly emerging technique of minimally invasive surgery [1], with notable value in cholecystectomy. It offers the advantage of better cosmetic results, less postoperative pain, and practically fewer postoperative infections, without jeopardizing the safety standards when compared with the conventional laparoscopic cholecystectomy (CLC) with four ports [2]. However, the use of single-port laparoscopic cholecystectomy (SLC) is met with apprehensions by the operating surgeons for being a difficult and stressful procedure, with no major difference in the total wound size compared to that of CLC [3].

The transumbilical approach was developed for SLC to reduce operative trauma and improve the cosmetic result following laparoscopic cholecystectomy [4]. However, this can often lead to an external collision of hands or clashing of instruments as the working space is small [3]. Also, being a more complex procedure, it has been deemed suitable for only nonobese patients with no history of abdominal surgery; it is not considered suitable for the 
acutely inflamed gallbladder. Furthermore, it is perceived that the single-port surgery requires greater operative time than CLC, while this additional burden of medical costs and safety remain undetermined $[5,6]$.

A newer approach, i.e., gasless laparoscopic surgery, has been performed using the abdominal wall-lifting method, which is now employed for various surgeries [7]. The abdominal wall lifting method allows the use of conventional reusable surgical instruments and valveless trocars because airtightness is not necessary during manipulations [8]. We have devised an alternative approach for laparoscopic cholecystectomy which requires inserting a single port at the umbilicus and using the abdominal wall-lifting method, eliminating the need to establish pneumoperitoneum. This article presents a comprehensive description of the procedure along with a retrospective analysis of the postoperative patient outcomes following the gasless SLC.

\section{MATERIALS AND METHODS}

This retrospective study is based on the medical records of patients undergoing laparoscopic cholecystectomy between December 2015 and December 2017 at Shikoku Central Hospital (Shikokuchuo, Japan). Data for 130 patients were obtained, of which 61 had undergone the gasless SLC, while the remaining 69 underwent the CLC upon the surgeons' preference. Patients with an acute severe or moderate cholecystitis, previous upper abdominal surgery, body mass index (BMI) more than $28 \mathrm{~kg} / \mathrm{m}^{2}$, and those with suspected malignancy on preoperative imaging were excluded from the study. All patients underwent abdominal ultrasonography and drip-infusion computed tomographic cholangiography preoperatively.

Demographic details (age, sex, BMI), history of pancreatitis, malignant disease, serum albumin level, coexisting diseases such as diabetes mellitus, hypertension, American Society of Anesthesiologists (ASA) physical status (PS) classification, and steroidsuse were collected and evaluated as preoperative characteristics.

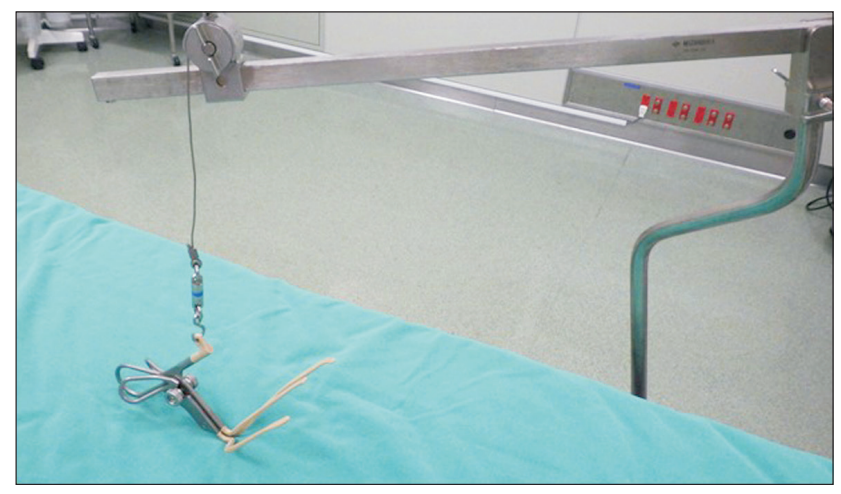

Fig. 1. A fan-shaped retractor is shown.
Duration of operation and postoperative hospital stay, postoperative complications, conversion to open or CLC, and drain use were also documented for analysis. Intravenous acetaminophen was routine use until 24 hours postoperatively, and afterward, oral nonsteroidal anti-inflammatory drugs were provided in all patients. Postoperative diet was provided on postoperative day 1 according to the clinical pathway of both groups.

\section{Surgical procedures}

Patients were placed in the lithotomy position, a 2- to 3-cm vertical transumbilical incision was made, and an Alexis wound retractor (Applied Medical, Rancho Santa Margarita, CA, USA) was inserted into the abdomen without difficulty as one port. The Alexis wound retractor essentially creates trocar positions with flexible fulcrums that allow combined motions in linear, radical, and translational planes. The larger the incision becomes, the greater the movement between the instruments. Abdominal distension was attained using a fan-shaped retractor (Mizuho, Tokyo, Japan) without the use of carbon dioxide insufflation (Fig. 1). A 5-mm flexible scope, one modified curved grasper, one dissector, and a part of the traditional laparoscopic instrument set were used to give the feeling of three dimensions during dissection (Fig. 2). An endoractor (Kawamoto, Tokyo, Japan) was inserted via one port to exert pressure on the duodenum and colon. The endoractor is a sponge that is $24 \times 1 \mathrm{~cm}$ in size when dry and increases in weight and size by absorbing fluid (Fig. 3).

Next, the gallbladder was retracted at the fundus and approached from the Calot triangle. The cystic duct and choledochus were identified with a grasper while retracting the fundus, and the cystic duct and artery were partially exposed. A right angle-type dissector was used to expose the cystic duct and

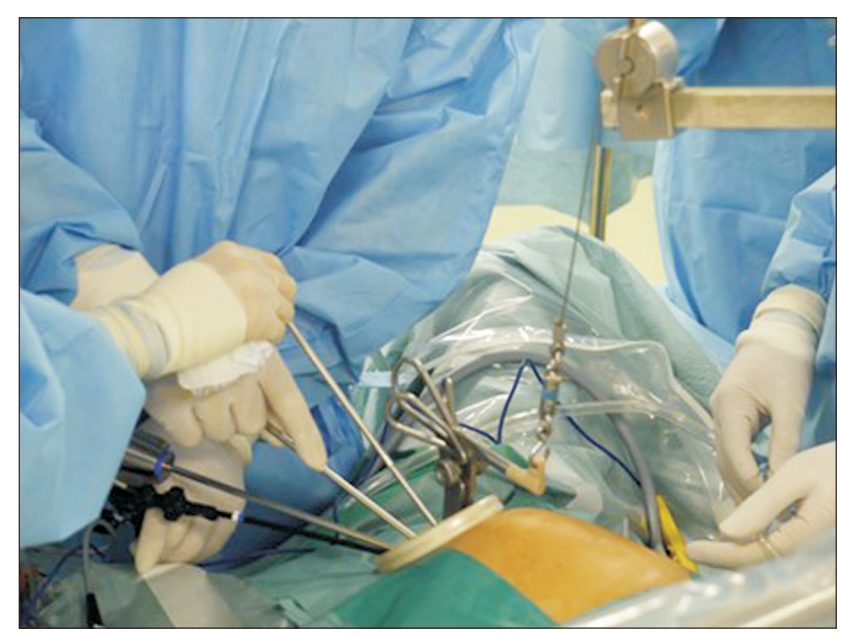

Fig. 2. One grasper and two dissectors were used through one port in the umbilicus. 


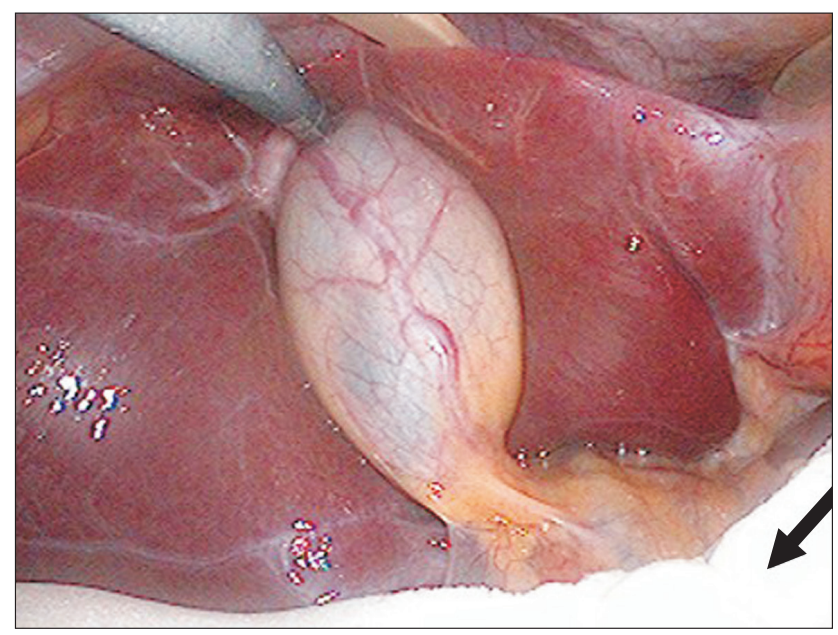

Fig. 3. An endoractor pointed by the arrow was used to obtain a good view by oppression. This thin sponge is inserted through the umbilical port and is augmented by sprinkling saline solution.

artery completely. For patients in whom adequate exposure to the Calot triangle was achieved, the cystic artery and duct were ligated with 3-0 polyglycolic acid suture or clipped and divided. The choice of ligation with 3-0 polyglycolic acid suture or use of surgical clip was made based on the surgeon's preference. Thereafter, the gallbladder was pushed upright and released from the liver bed using the hook monopolar cautery. In patients in whom the cystic duct and artery were not easily identifiable, retrograde cholecystectomy was performed from the fundus of the gallbladder. Finally, the gallbladder was separated from the liver bed, and the specimen was removed through the umbilical port.

\section{Statistical analysis}

All quantitative data are expressed as mean \pm standard deviation. The patient characteristics, intraoperative, and postoperative factors of both SLC and CLC groups were compared using the chi-square test and the Student $t$ test as appropriate. The $p$ value of $<0.05$ was considered for statistical significance. All analyses were performed using the software package IBM SPSS version 20.0 (IBM Corp., Armonk, NY, USA).

\section{RESULTS}

The SLC group $(\mathrm{n}=61)$ consisted of 25 male and 36 female patients with a mean age of $58 \pm 7$ years (range, 35-81 years), while the CLC group $(\mathrm{n}=69)$ comprised 28 males and 41 females with a mean age of $53 \pm 8$ years (range, 38-83 years) (Table 1). Both groups were similar in terms of BMI of the patients (SLC group, $23 \pm 3 \mathrm{~kg} / \mathrm{m}^{2}$ and CLC group, $23 \pm 2 \mathrm{~kg} / \mathrm{m}^{2}$; range, $18-28 \mathrm{~kg} / \mathrm{m}^{2}$ ). Regarding the indication for cholecystectomy, the patients un-
Table 1. Patient demographics between study arms

\begin{tabular}{lccc}
\hline \multicolumn{1}{c}{ Variable } & $\begin{array}{c}\text { SLC group } \\
(\mathrm{n}=61)\end{array}$ & $\begin{array}{c}\text { CLC group } \\
(\mathrm{n}=69)\end{array}$ & $p$ value \\
\hline Age (yr) & $58.1 \pm 7.2$ & $53.4 \pm 8.6$ & 0.49 \\
Sex, male/female & $25 / 36$ & $28 / 41$ & 0.15 \\
Body mass index (kg/m²) & $23.1 \pm 3.2$ & $23.5 \pm 2.0$ & 0.59 \\
\hline Disease, stone/polyp & $56 / 5$ & $65 / 4$ & 0.20 \\
Previous surgery (\%) & $8(13.1)$ & $5(7.2)$ & 0.27 \\
\hline ASA PS classification, & $57 / 3 / 1$ & $61 / 6 / 2$ & 0.79 \\
$\quad$ I \& I/III/IV & & & \\
Serum albumin level (g/dL) & $4.6 \pm 0.6$ & $4.5 \pm 0.4$ & 0.26 \\
\hline Diabetes mellitus & $9(14.8)$ & $10(14.5)$ & 0.97 \\
Hypertension & $12(19.7)$ & $18(26.1)$ & 0.37 \\
COPD & $4(6.6)$ & $5(7.2)$ & 0.78 \\
Steroids use & $1(1.6)$ & $2(2.9)$ & 0.63 \\
\hline Previous cholecystitis & $8(13.1)$ & $3(4.3)$ & 0.64 \\
\hline Previous pancreatitis & $2(3.2)$ & $3(4.3)$ & 0.75 \\
\hline
\end{tabular}

Data are expressed as mean \pm standard deviation, number only, or number (\%).

SLC, single-port laparoscopic cholecystectomy; CLC, conventional gasless laparoscopic cholecystectomy; ASA, American Society of Anesthesiologists; PS, physical status; COPD, chronic obstructive pulmonary disease.

Table 2. Comparing intraoperative and postoperative characteristics between study arms

\begin{tabular}{lccc}
\hline \multicolumn{1}{c}{ Variable } & $\begin{array}{c}\text { SLC group } \\
(\mathrm{n}=61)\end{array}$ & $\begin{array}{c}\text { CLC group } \\
(\mathrm{n}=69)\end{array}$ & $p$ value \\
\hline Operative time (min) & $64.5 \pm 18.5$ & $58.6 \pm 17.3$ & 0.06 \\
\hline Operative blood loss (mL) & $12.0 \pm 10.1$ & $10.4 \pm 5.1$ & 0.16 \\
Additional port & 2 & 0 & 0.18 \\
Drain used & $14(23.0)$ & $52(72.4)$ & 0.001 \\
Complication & 0 & 2 & 0.64 \\
$\quad$ Surgical site infection & 0 & 1 & \\
Biliary duct injury & 0 & 1 & \\
Conversion to open & 0 & 2 & 0.13 \\
Analgesic dose (time) & $2.5 \pm 2.0$ & $3.1 \pm 2.3$ & 0.12 \\
Hospital stay period (day) & $4.5 \pm 2.1$ & $4.8 \pm 4.1$ & 0.31 \\
Oral feeding time (day) & $1.1 \pm 0.5$ & $1.2 \pm 0.2$ & 0.21 \\
\hline
\end{tabular}

Data are expressed as mean \pm standard deviation, number only, or number (\%).

SLC, single-port laparoscopic cholecystectomy; CLC, conventional gasless laparoscopic cholecystectomy. 
dergoing both SLC and CLC had a similar incidence of gallbladder stones (92\% and 94\%) and gallbladder polyps (8\% and 6\%), respectively. Overall, the two groups were comparable in terms of mean age, sex, disease, ASA PS classification, and comorbidity.

When comparing the mean duration of the surgery, those of the two groups were not different as against the perception about SLC of being longer (SLC, $64.5 \pm 18.5$ minutes vs. CLC, $58.6 \pm$ 17.3 minutes, $p=0.06$ ) (Table 2). Likewise, almost all postoperative variables (the frequency of analgesic use, postoperative stay, postoperative complications, and blood loss), except for drain use, showed no statistically significant differences between the two groups. Patients with acute severe or moderate cholecystitis were excluded from the study. However, eight patients in the CLC group and three patients in the SLC group had previous mild cholecystitis. There was no significant difference in the rate of previous cholecystitis and pancreatitis. In the two groups, no significant difference was shown in previous lower abdominal surgeries such as appendectomy, resection of the intestine, and hysterectomy. In most patients of the CLC group, drain use was chosen. This was done based on the surgeon's preference but had no effect on postoperative hospital stay. Analgesic doses of intravenous acetaminophen used postoperatively showed no significant difference in the two groups.

While the average time taken to complete the SLC procedure in the first five patients in this group was $98.2 \pm 20.0$ minutes (range, 65-140 minutes), the other 56 required only $58.3 \pm 13.1$ minutes (range, 35-120 minutes; $p<0.01$ ). No patient required conversion to open surgery. Also, additional placement of trocars in a more lateral position was required in two patients to control bleeding at the liver bed or the gallbladder and give a better laparoscopic view. There was no instance of the gallbladder being punctured and drained to facilitate traction, and no gallbladder specimens were lost intraoperatively. Finally, no postoperative complications were encountered.

\section{DISCUSSION}

This study describes the transumbilical approach used for SLC, which is a recent laparoscopic procedure and described by some authors as 'scarless' [3,9]. The existing literature rationalizes the clinical significance of SLC in terms of cosmetic value and early relief from postoperative pain associated with this surgery. However, there is a lack of advanced evidence (level I and II) addressing the concerns related to its operative outcomes [10]. As a fact, a systematic review comparing SLC and CLC by Evers et al. [11] reported that SLC does not offer any significant advantages over CLC other than reduced postoperative pain and better cosmesis. However, with the advancements and learning in the field of minimally invasive surgeries, the technique for SLC has improved, and newer devices have been developed. The latest addi- tion, a Triport (Olympus Corp., Tokyo, Japan) laparoscopic access device, allows multiple instruments to pass through one incision at the same time and simultaneously ensure pneumoperitoneum $[12,13]$. However, such operations have no port to evacuate the smoke created by cautery. Alternatively, we used the abdominal wall-lifting method to establish single-port access, which has reduced some of the technical challenges faced during SLC. To the best of our knowledge, the present report documents the first clinical experience of a gasless SLC using transumbilical access.

Compared to the conventional method to create a pneumoperitoneum, the gasless technique offers several advantages like allowing an increased range of motion, dexterity, and stability of the scope and instruments, along with diminishing the chances of clashing and the associated operative costs. However, the main disadvantage of SLC is the longer operating time required compared to the CLC method [14].

This may be related to the technical hitches associated and a steep learning curve required to master the skill. However, the present study remarkably demonstrates that the mean operating time reduced from an average of 98.2 minutes in the first five SLC surgeries to 58.3 minutes in the latter 56 surgeries. Based on these data, it can be reasoned that the learning period for the present method is short, with operating time being comparable time to the CLC.

A beginner may also be aided in learning by the recent technical improvements that ensure wider utilization of the single-port surgery. We used an Alexis wound retractor instead of the specially designed single-entry ports, which are costly and difficult to use because of the less maneuverability offered. This wound retractor used as a flexible valveless fulcrum allows the introduction of three to five instruments, with minimal clashing, while maximizing the freedom of manipulations. Additionally, the use of an endoractor to apply pressure on the intestines improves the laparoscopic view. The operative view obtained on the monitor was almost as good as with CLC. We did not use additional puncture wounds for suture slings or a mini loop retractor since there was adequate access to appropriately retract the gallbladder. Consequently, this method widens the activity range of both the operator and the endoscopist, besides mitigating the difficulties associated with the pneumoperitoneum method.

Nevertheless, there are several technical difficulties related to the performance of single-port surgery. Kim et al. [13] recommended the fundus-first laparoscopic cholecystectomy as a single-port method. This is in contrast to the more commonly used method of blunt dissection, beginning around the cystic duct and artery, and using the grasper to retract the gallbladder laterally. We customarily performed a retrograde cholecystectomy in traditional laparoscopic cholecystectomy, while the fundus-first laparoscopic cholecystectomy can be performed in patients with an inflamed gall bladder. 
Another important criticism in the change to single-port surgery is the potentially impaired view of the Calot triangle caused by the in-line instruments and scope [15]. However, there are some clashing and overlapping in the pneumoperitoneum method as well. Coordination between the right and left-hand instruments is essential to develop dexterity since the left hand is used for retracting the gallbladder, while the right hand is used for dissection. Moreover, it is advisable to avoid contact between the operative instruments and the camera, as the use of crossedover articulating instruments requires a longer operative time to achieve careful and precise dissection, and lesser force is available for tissue dissection than with the CLC technique [15]. To counter this, we developed an easy method of manipulating the instruments through one port. The valveless port, $3 \mathrm{~cm}$ in diameter, allows the same execution of the instruments with both hands as with the traditional four-port method. This wound retractor allows easy handling of instruments and scopes of various sizes without requiring an additional port to retract the gallbladder. Hence, the gasless SLC is feasible, and no significant differences with the CLC were observed in terms of postoperative complications.

The outcomes with SLC could possibly be better not only regarding the better quality of care in terms of pain, cosmesis, and safety of the patients but also medical costs. Pain is the most frequent complaint of patients undergoing laparoscopic cholecystectomy in the early postoperative stage. The prognosis of postoperative pain with SLC has been controversial [14]. However, in the present study, no significant decrease in postoperative pain was seen in patients of SLC when compared to CLC.

Still, as this is a retrospective study, future studies should undertake randomized control trials to verify these results. SLC, using the abdominal wall-lifting method, represents a more costeffective procedure over the conventional pneumoperitoneum technique since most instruments used in the former are reusable [16]. Also, this method does not require the use of a Triport, other ports, or clip-appliers. A previous study in this series conducted in the same hospital showed that the costs of the operative equipment used in CLC using the abdominal wall-lifting method were $\$ 85 \pm \$ 10$, vs. $\$ 525 \pm \$ 50$ for the pneumoperitoneum method using disposable instruments [17]. Besides the additional costs of operative instruments and increased difficulty due to instrument clashing, pneumoperitoneum leak has been described as a real disadvantage of single-port surgery using this method [18].

Moreover, single-port surgery gives less than adequate visualization of Calot triangle in patients with considerable gallbladder inflammation or obesity [17]. In such cases, it may be better to add a mini loop retractor or extra ports for fundus or liver retraction, with no need for a change in operative position. Also, surgeons often face difficulty in inserting a 10-mm clip-applier on the cystic duct and artery. We overcame this problem by in- serting right-angled instruments and tying the cystic duct and artery. Therefore, greater experience and skill are required to perform single-port surgery in case of difficult laparoscopic cholecystectomy. Undeniably, the patients selected to be studied in the series had BMI corresponding to normal height-weight with only mild inflammation of the gallbladder. Therefore, improved instruments, particularly articulating graspers and dissectors, may further decrease the difficulty level $[13,19]$. Sizable studies are needed to understand whether the present method can decrease pain scores and provide better cosmetic results over traditional laparoscopic or pneumoperitoneum SLC.

In conclusion, SLC with abdominal wall lifting shows comparable procedural and postoperative outcomes when compared to the traditional four-port laparoscopic cholecystectomy. It can be used with traditional laparoscopic instruments with a few simple modifications. Our new method not only produces minimal postoperative discomfort and no additional scars but is costeffective as well. In contrast to the current evidence indicating increased costs of SLC with pneumoperitoneum, a reduction in the use of disposable operative materials was seen with a short learning curve required to master the procedure. However, early conversion to the traditional method is recommended to avoid severe complications when technical difficulties are encountered.

\section{NOTES}

\section{Ethical statements}

We conducted this study in compliance with the principles of the Declaration of Helsinki. The study's protocol was reviewed and approved by the Institutional Review Board of Shikoku Central Hospital (No.3-3). Written informed consent from patients was waived because of the retrospective nature of this study.

\section{Funding/support}

None.

\section{Authors' contributions}

Conceptualization, Investigation, Visualization: MI

Data curation: YT

Formal analysis: MI, MA

Methodology: MI, YT

Project administration: KM

Writing-original draft: MI, HT, KM

Writing-review \& editing: MI, TM

All authors read and approved the final manuscript. 


\section{Conflict of interest}

All authors have no conflicts of interest to declare.

\section{ORCID}

Masashi Ishikawa, https://orcid.org/0000-0001-5434-6443

Michihito Asanoma, https://orcid.org/0000-0001-5827-9188

Yoshihiko Tashiro, https://orcid.org/0000-0002-7136-275X

Hirokazu Takechi, https://orcid.org/0000-0002-9337-1438

Kazuo Matsuyama, https://orcid.org/0000-0002-2635-4080

Takayuki Miyauchi, https://orcid.org/0000-0002-8609-9798

\section{REFERENCES}

1. Romanelli JR, Mark L, Omotosho PA. Single port laparoscopic cholecystectomy with the TriPort system: a case report. Surg Innov 2008; 15:223-228.

2. Scott DJ, Tang SJ, Fernandez R, et al. Completely transvaginal NOTES cholecystectomy using magnetically anchored instruments. Surg Endosc 2007;21:2308-2316.

3. Cinar H, Topgul K, Malazgirt Z, et al. Early results of single-incision laparoscopic cholecystectomy in comparison with the conventional: does it have any impact on quality of life? Ann Med Surg (Lond) 2018;32:1-5.

4. Cuesta MA, Berends F, Veenhof AA. The "invisible cholecystectomy": a transumbilical laparoscopic operation without a scar. Surg Endosc 2008;22:1211-1213.

5. Langwieler TE, Nimmesgern T, Back M. Single-port access in laparoscopic cholecystectomy. Surg Endosc 2009;23:1138-1141.

6. Malik M, McCormack K, Krukowski ZH, et al. Single port/incision laparoscopic surgery compared with standard three-port laparoscopic surgery for appendicectomy: a randomized controlled trial. Trials 2012;13:201.

7. Ishikawa M, Tagami Y, Toyota T, et al. An initial training of laparoscopic cholecystectomy: the abdominal wall lifting vs. pneumoperitoneum procedures. Int J Surg Invest 2001;3:421-426.

8. Hyodo M, Sekiguchi C, Ohdaira T, Chiba H, Ohtsuka S, Nagai H.
Gasless laparoscopy-assisted colon surgery utilizing mini-laparotomy and a subcutaneous abdominal wall lift system. Hepatogastroenterology 2004;51:1011-1014.

9. Tyagi S, Sinha R, Tyagi A. Single incision laparoscopic cholecystectomy: less scar, less pain. J Minim Access Surg 2017;13:118-123.

10. Casaccia M, Palombo D, Razzore A, Firpo E, Gallo F, Fornaro R. Laparoscopic single-port versus traditional multi-port laparoscopic cholecystectomy. JSLS 2019;23:e2018.00102.

11. Evers L, Bouvy N, Branje D, Peeters A. Single-incision laparoscopic cholecystectomy versus conventional four-port laparoscopic cholecystectomy: a systematic review and meta-analysis. Surg Endosc 2017;31:3437-3448.

12. Merchant AM, Cook MW, White BC, Davis SS, Sweeney JF, Lin E. Transumbilical Gelport access technique for performing single incision laparoscopic surgery (SILS). J Gastrointest Surg 2009;13:159-162.

13. Kim HJ, Lee JI, Lee YS, et al. Single-port transumbilical laparoscopic appendectomy: 43 consecutive cases. Surg Endosc 2010;24:2765-2769.

14. Sunamak O, Donmez T, Ferahman S, Uludag SS, Avaroglu HI. A promising technique for easier single incision laparoscopic cholecystectomy: needle grasper traction of gallbladder. Wideochir Inne Tech Maloinwazyjne 2018;13:358-365.

15. Tacchino R, Greco F, Matera D. Single-incision laparoscopic cholecystectomy: surgery without a visible scar. Surg Endosc 2009;23:896899.

16. Borle FR, Mehra B, Ranjan Singh A. Comparison of cosmetic outcome between single-incision laparoscopic cholecystectomy and conventional laparoscopic cholecystectomy in rural Indian population: a randomized clinical trial. Indian J Surg 2015;77(Suppl 3):877-880.

17. Ishikawa $M$, Nakagawa $T$, Nishioka $M$, et al. Costs and benefits of laparoscopic cholecystectomy: abdominal wall lifting vs. pneumoperitoneum procedure. Hepatogastroenterology 2006;53:497-500.

18. Rao PP, Bhagwat SM, Rane A, Rao PP. The feasibility of single port laparoscopic cholecystectomy: a pilot study of 20 cases. HPB (Oxford) 2008;10:336-340.

19. Pluchino N, Buchs NC, Drakopoulos P, Wenger JM, Morel P, Dällenbach P. Robotic single-site combined cholecystectomy and hysterectomy: advantages and limits. Int J Surg Case Rep 2014;5:1025-1027. 